\title{
Preliminary study of the optimization of protocol for development of type 2 diabetic model in rats
}

\begin{abstract}
The existence of experimental animal model helps in the understanding of pathopysiology of diabetes and facilitates in the development of drugs for its treatment. Chemical induction using streptozotocin (STZ) shows to be the most popularly used procedure in induction of diabetes in experimental animals. Numerous studies have shown that development of diabetic animal models using certain dosage of STZ on Wistar and Sprague Dawley rats. The aim of the present investigation was conducted to develop type 2 diabetic model using double dose of nicotinamide (NA) and STZ on Sprague Dawley rats within 7 days. Male Sprague-Dawley (250-280 g) were injected with NA, 15 minutes prior the injection of STZ via single and double dose of intraperitoneal (i.p) injection, after overnight fasting. The blood glucose level was monitored from the diabetic animal on day 3, 7, 14 and 21 after the induction of diabetes. Blood glucose levels $>11.0 \mathrm{mmol} / \mathrm{L}$ were considered as diabetic condition. In addition, physiological parameters such as food and fluid intakes, changes in body weight and biochemical parameters, blood glucose level were compared with diabetic and control group. In conclusion, the chemically induced diabetic model in Sprague Dawley rats appears to be not suitable compared to the other experimental model which using high fat diet (HFD) and low dose of streptozotocin.
\end{abstract}

Keyword: Streptozotocin-nicotinamide induced diabetes; Sprague dawley; Type 2 diabetes 